\title{
A TDDFT Study of the Excited States of DNA Bases and Their Assemblies
}

\author{
Daniele Varsano, ${ }^{*, \dagger, *}$ Rosa Di Felice, ${ }^{\S}$ Miguel A. L. Marques,,$+\| l$ and Angel Rubio ${ }^{\dagger, \ddagger, \perp}$ \\ Departamento de Física de Materiales, Facultad de Quimicas, Universidad del País Vasco and Donostia \\ International Physics Center (DIPC), 20080 San Sebastian, Spain, European Theoretical Spectroscopy Facility \\ (ETSF), National Center on nanoStructures and Biosystems at Surfaces (S3) of INFM-CNR, Via Campi 213/A, \\ 41100 Modena, Italy, Centro de Física Computacional, Departamento de Física da Universidade de Coimbra, \\ Rua Larga, 3004-516 Coimbra, Portugal, and Institut für Theoretische Physik, Freie Universität Berlin, \\ Arnimallee 14, D-14195 Berlin, Germany
}

Received: October 25, 2005; In Final Form: February 21, 2006

\begin{abstract}
We present a detailed study of the optical absorption spectra of DNA bases and base pairs, carried out by means of time dependent density functional theory. The spectra for the isolated bases are compared to available theoretical and experimental data and used to assess the accuracy of the method and the quality of the exchangecorrelation functional. Our approach turns out to be a reliable tool to describe the response of the nucleobases. Furthermore, we analyze in detail the impact of hydrogen bonding and $\pi$-stacking in the calculated spectra for both Watson-Crick base pairs and Watson-Crick stacked assemblies. We show that the reduction of the UV absorption intensity (hypochromicity) for light polarized along the base-pair plane depends strongly on the type of interaction. For light polarized perpendicular to the basal plane, the hypochromicity effect is reduced, but another characteristic is found, namely a blue shift of the optical spectrum of the base-assembly compared to that of the isolated bases. The use of optical tools as fingerprints for the characterization of the structure (and type of interaction) is extensively discussed.
\end{abstract}

\section{Introduction}

Optical absorption and circular dichroism are widely used experimental tools to characterize the structural and dynamical properties of biomolecular systems. The particular merit of optical characterization tools is that they can discriminate between intrinsic molecular properties and solvent-induced effects. This is especially important for DNA and DNA-based compounds. $^{1-3}$ Traditionally, DNA molecules have always retained a special place in scientific investigation, for biological/ medical issues. Lately, in the field of nanotechnology, DNA is also attracting interest for several potential applications due to its stability (in solution), to its one-dimensional character, and to the regular $\pi$-stacking, along with the unique properties of self-assemby and recognition. ${ }^{4-8}$ In relation to nanotechnology exploitation, the determination and interpretation of the electronic properties of nucleobases and of DNA helical arrangements are an extremely valuable foreword, and notable multidisciplinary efforts are currently devoted to such goals: we refer the reader to recent reviews about electronic structure calculations and possible charge motion behaviors. ${ }^{4,9}$ Furthermore, knowledge of the electronic properties, excited-state lifetimes, and ultraviolet (UV) absorption spectra is of paramount importance for our understanding of, e.g., the crucial phenomenon of UV radiation-induced DNA damage. ${ }^{10-12}$ From this brief preamble, it is clear that the full characterization of the optical properties of DNA molecules and DNA-based complexes is of great interest. To relate the optical properties of the nucleic

\footnotetext{
* Corresponding author.

†niversidad del País Vasco and DIPC.

European Theoretical Spectroscopy Facility.

$\S$ National Center on nanoStructures and Biosystems at Surfaces.

"Universidade de Coimbra.

${ }^{\perp}$ Freie Universität Berlin.
}

acids to their structure, spatial conformation, and type of intramolecular interactions, a valuable preliminary step is to gain insight into the excited-state properties of their building blocks, namely the monomeric bases, and to understand the role of hydrogen-bonding and stacking when these monomeric units form complex assemblies. In their natural environment, the DNA bases are paired via hydrogen-bonds in the Watson-Crick scheme, ${ }^{13}$ and are covalently bonded to the sugar-phosphate backbone. The hydrogen-bonded base pairs interact with each other in the typical helical arrangement by interplane van der Waals forces. ${ }^{14}$ To disentangle how the different interactions control the DNA dynamics upon light absorption, it is important to infer how the spectrum of a given isolated nucleobase is modified by mutual interactions in the different spatial conformations of DNA assemblies. This is the goal of the present work: to provide a systematic study of the stacking and $\mathrm{H}$-bonding interaction effects in the optical spectra of molecular complexes formed with isolated nucleobases. We undertake this task by means of time-dependent-density functional theory (TDDFT) calculations. ${ }^{15-18}$

TDDFT is gaining increasing popularity as an efficient tool to calculate electronic excitations in finite systems, thanks to its simplicity and moderate computational cost. Since the very beginning, TDDFT has provided good results for the optical response of a large set of molecular systems, ${ }^{19}$ including some biomolecules (e.g., green fluorescent protein chromophores and their mutants, ${ }^{20,21}$ chlorophylls, ${ }^{22,23}$ flavins, ${ }^{24}$ and nucleic acid bases, ${ }^{25,26}$ among many others). It is still perhaps premature to discuss the general level of accuracy of TDDFT when applied to biomolecules, especially when dealing with van der Waals complexes. ${ }^{27}$ Furthermore, as the field is quite recent, we can still expect rapid methodological developments, in particular toward the derivation of better exchange-correlation functionals. 
However, the existing results are already promising and pave the way for a broader application of TDDFT in biochemistry.

Before studying complex helical DNA-based biopolymers, extensive tests are required to prove the performance and predictive power of TDDFT for DNA-based systems. One of the aims of the present work is to provide results from firstprinciples calculations of simple DNA-based assemblies that can be used as reference for future developments and studies. This reference set includes the isolated DNA bases, hydrogen bonded Watson-Crick pairs, a stacked guanine-cytosine dimer, and a quartet formed by two stacked guanine-cytosine pairs.

For the isolated DNA bases there are plenty of experimental and theoretical results concerning their optical response. ${ }^{2}$ We do not carry out a full systematic analysis of all the published data for the isolated DNA bases, but only those more pertaining to our investigation. The computational tools applied to nucleobases range from single excitation configuration interaction (CIS), to complete active space second-order perturbation theory (CASPT2), and include other approaches such as multireference perturbation configuration interaction (CIPSI). A review of the performance of such methods on the nucleobases, ${ }^{2}$ as well as relevant experimental data can be found in refs $28-30$.

Despite the large number of works on the excited states of isolated DNA bases, a limited number of studies is devoted to base pairs and base assemblies due to their complexity. Shukla and Leszczynski studied adenine-uracil, ${ }^{31}$ adenine-thymine (AT), and guanine-cytosine (GC) pairs in the Watson-Crick configuration $^{32}$ at the CIS level; Sobolewski and Domcke ${ }^{33}$ studied the low lying energy part of the spectrum of GC base pairs with the more sophisticated CASPT2 technique; Wesolowski ${ }^{34}$ used an embedding method to study the lowest excited state of the GC and AT pairs. Very recently, Tsolakidis and Kaxiras $^{25}$ computed the whole absorption spectra of the GC and AT pairs in different tautomeric forms of the bases in the TDDFT framework.

Moreover, very few studies exist on the excited-state properties of bases in a stacked configuration. Jean and Hall studied the fluorescent properties of dimers of 2-aminopurine stacked with DNA bases ${ }^{35}$ in different forms and stacked trimers containing 2-aminopurine, ${ }^{36}$ showing the relevance of the stacked geometries in the character of the excited-state transitions. To the best of our knowledge, the present results are the first $a b$ initio calculations dealing with stacked natural pairs. Yet, we remark that the present results are for free-standing nucleobase complexes, i.e., not including solvation effects. These effects are known to be more important for $n \pi^{*}$ than $\pi \pi^{*}$ type transitions. ${ }^{29,32,37-40}$

The paper is organized as follows. In section 2 we provide the details of the real-time real-space method ${ }^{41}$ used to compute the optical properties. In section 3 we present our results for the isolated gas-phase nucleobases, i.e., guanine, cytosine, adenine, thymine, and uracil, ( $\mathrm{G}, \mathrm{C}, \mathrm{A}, \mathrm{T}$, and $\mathrm{U}$, respectively). To understand the role of hydrogen-bonding in shifting and modifying the spectral features, we show, in section 4 , results for H-bonded Watson-Crick GC and AT base pairs (labeled $\mathrm{GC}_{\mathrm{H}}$ and $\mathrm{AT}_{\mathrm{H}}$, respectively). In section 5 we present results for a $\mathrm{GC}$ stacked dimer (labeled $\mathrm{GC}_{\mathrm{S}}$ ) that mimics the arrangement between $\mathrm{C}$ and $\mathrm{G}$ in two consecutive planes in the real DNA double helix. We also discuss the relative roles of $\pi$-stacking and hydrogen bonding in the optical absorption of a stacked quartet made of two adjacent Watson-Crick GC pairs as in A-DNA [labeled d(GC)], that combines hydrogen-bonding and $\pi$-stacking. In section 6 we summarize the results of the present work and provide some perspectives for future studies.

\section{Computational Framework}

2.1. Optical Spectra by TDDFT. The optical absorption spectra of DNA bases and base pairs were computed within a real-space real-time version of TDDFT, as implemented in the code octopus. ${ }^{41}$ This method does not rely on perturbation theory and is competitive with implementations in the frequency domain. ${ }^{42-45}$ The theoretical background and the computational details of our scheme are extensively described elsewhere: $41,42,46,47$ here we just summarize the crucial aspects relevant to the present study of DNA complexes.

The starting point is the calculation of the ground state electronic structure in the DFT framework, which is done within a pseudopotential approach (see below for details). To access the excited state properties, the ground state is then instantaneously perturbed with an electric field of magnitude $k_{0}$ along the three principal Cartesian directions (i.e., by applying the external potential $v(\boldsymbol{r}, t)=-k_{0} x_{v} \delta(t)$, where $\left.x_{v}=x, y, z\right)$. The amplitude $k_{0}$ must be sufficiently small in order to keep the system dipolar response linear. In this impulsive approach, all the frequencies of the system are excited with the same weight. Next, the time-dependent Kohn-Sham (KS) orbitals are propagated for a finite time, and the dynamical polarizability is obtained as

$$
\alpha_{v}(\omega)=-\frac{1}{k_{0}} \int \mathrm{d}^{3} r x_{v} \delta n(\boldsymbol{r}, \omega)
$$

where $\delta n(\boldsymbol{r}, \omega)$ is the Fourier transform of the relative density $n(\boldsymbol{r}, t)-n_{0}(\boldsymbol{r})$, and $n_{0}(\boldsymbol{r})$ stands for the ground state density. The photoabsorption cross-section, the quantity usually measured in experiments, is proportional to the imaginary part of the polarizability averaged over the three space directions

$$
\sigma(\omega)=\frac{4 \pi \omega}{c} \frac{1}{\mathcal{F}} \sum_{v} \alpha_{v}(\omega)
$$

where $c$ is the velocity of light. Another widely used quantity is the dipolar strength function $S_{v}(\omega),{ }^{18,41}$ which is connected to $\alpha_{\nu}(\omega)$ by

$$
S_{v}(\omega)=\frac{2 m}{\pi \mathrm{p}^{2}} \mathscr{F}_{v}(\omega)
$$

With this definition, the Thomas-Reiche-Kuhn sum rule integrates to the number of electrons in the system. $S_{\nu}(\omega)$ is the quantity plotted in our figures.

A significant advantage of this real-time approach is the fact that only occupied states are needed, thus avoiding the calculation of the unoccupied states that instead enter the traditional orbital (occupied-empty) representation of the linear response equation. ${ }^{48}$ The complete set of empty orbitals required in the latter approach is fully accounted for by the time propagation. In addition, it is important to note that in the time domain only the approximation to the exchange-correlation potential $v_{\mathrm{xc}}$ is required, whereas in conventional frequency-domain linear response TDDFT formulations the $f_{\mathrm{xc}}$ kernel is needed also. ${ }^{15-18,43-45}$ For $v_{\mathrm{xc}}$ we employed the local density approximation (LDA) with the Perdew-Zunger parametrization. ${ }^{49}$ In the case of the isolated guanine base, we also performed the time propagation with the generalized gradient approximation (GGA) PW91 ${ }^{50} v_{\mathrm{xc}}$ functional. As expected, ${ }^{51}$ no substantial differences were detected between the two parametrizations. Therefore, and as the LDA functional is numerically the most 


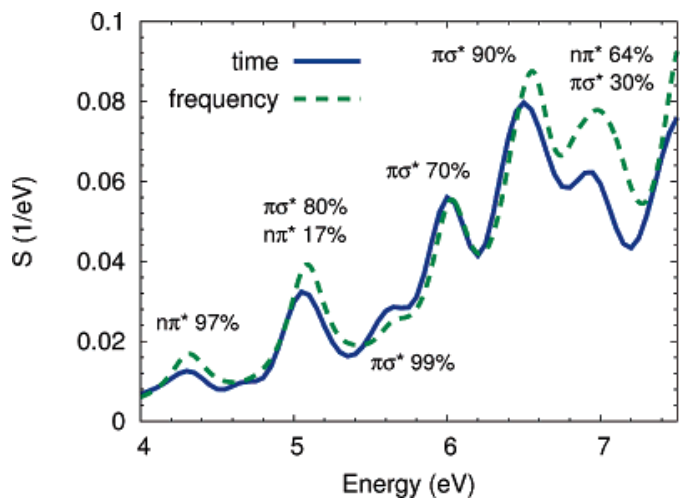

Figure 1. Dipole strength function of cytosine along the axis perpendicular to the base plane, calculated through the real-time propagation (blue solid curve) and in the frequency domain ${ }^{48}$ (green dashed curve). An artificial broadening of $0.15 \mathrm{eV}$ was adopted as an aid to the eye to compare time and frequency domain spectra. Note that we can distinguish the $n \pi^{*}$ character in an indisputable way only for the lowest energy peak. At higher energy the $n \pi^{*}$ character is less pure, due to the mixing of a large number of transitions. This fact is illustrated here for cytosine, but we observed it for all the investigated nucleobases.

stable, we chose to use this functional in the time propagation for all the other investigated systems. ${ }^{52}$

Concerning technical details: We used a real-space grid made of overlapping spheres with a radius of $4 \AA$ centered around each nucleus. All quantities were discretized in a uniform grid with spacing $0.23 \AA$. The time-step for the time evolution was $0.0066 \mathrm{fs}$ (which ensures stability in the time-dependent propagation of the KS wave functions) and the total propagation time was at least $20 \mathrm{fs}$. Such simulation parameters ensure wellconverged absorption spectra up to about $8 \mathrm{eV}$. The energy resolution, dictated by the total simulation length by $\Delta E \sim h / T$, is better than $30 \mathrm{meV}$. This sets the lower limit for the line width of the calculated spectra. The electron-ion interaction (both in the time-dependent and time-independent DFT calculations) was modeled by norm-conserving pseudopotentials. ${ }^{53}$

To compare with experiments where the molecules are not aligned with respect to the applied field, we need to average the response along the three Cartesian axes [cf. eq 2]. This result can be directly compared to other available data and used to discriminate among different nucleobase conformations. However, the analysis can be pushed even further: in the case of planar molecules (isolated bases, $\mathrm{GC}_{\mathrm{H}}, \mathrm{AT}_{\mathrm{H}}$ ), one can easily distinguish between in-plane and out-of-plane transitions. While the former are prevalently $\pi \pi^{*}$ and have large oscillator strengths, the latter are much weaker (by one or two orders of magnitude) and are usually hidden under the stronger signals. To unveil the character of the weak transitions, the time-domain analysis is not sufficient. Thus, we also performed linear response calculations in the frequency domain ${ }^{48}$ for all planar structures (isolated bases, $\mathrm{GC}_{\mathrm{H}}$, and $\mathrm{AT}_{\mathrm{H}}$ pairs). In this formalism, several pairs of occupied/unoccupied orbitals participate in each excitation with different weights: it is then possible to qualitatively assign to each excitation the predominant character of the contributing transitions. In Figure 1 we show an example of the transition character of spectral features, derived from our frequency analysis of cytosine. The dipole strength function of cytosine along the direction perpendicular to the base plane, obtained by both the time-domain and frequency-domain techniques, is plotted against energy. The agreement encountered between the two techniques turns out to be good in the accessible energy range. For all other planar systems, we present the analysis of the transition character obtained in a similar way (namely, by comparing time-domain and frequency-domain features), but without explicitly showing the figures for the spectra obtained by frequency-domain TDDFT.

We note that in the frequency domain the transitions are delta functions. To make a fair comparison with the time-domain propagation, we have artificially broadened both spectra in Figure 1 using a Lorentzian function of $0.15 \mathrm{eV}$ width. Note that for the rest of the spectra shown in the paper obtained with the time-propagation scheme, we did not add any additional broadening and the resolution of $30 \mathrm{meV}$ is fixed by the duration of the time evolution. Any additional broadening comes from the coupling of the excited state to other excitations (e.g., Landau damping).

2.2. Ground-State Equilibrium Configuration by DFT. $a$. Starting Atomic Configurations. The starting atomic coordinates of the isolated DNA bases and of the $\mathrm{AT}_{\mathrm{H}}$ pair were derived from standard structural parameters for DNA,${ }^{54}$ whereas for the systems $\mathrm{GC}_{\mathrm{H}}, \mathrm{GC}_{\mathrm{s}}$, and $\mathrm{d}(\mathrm{GC})$ we extracted the simulated fragments from a high-resolution X-ray crystal structure of A-DNA, ${ }^{55}$ after a classical force-field structural adjustment. The crystal is formed by short helices of double stranded DNA with the sequence d(AGGGGCCCCT), a model for poly(dG)-poly(dC) DNA. The $\mathrm{H}$ coordinates (absent in the X-ray PDB file) were assigned using the HBUILD command ${ }^{56}$ of the CHARMM package. ${ }^{57,58}$ The CHARMM force field was also used for a raw relaxation of the entire d(AGGGGCCCCT) structure. From this grossly relaxed polymer we extracted the H-bonded and stacked GC fragments, which have been subsequently relaxed by quantum simulations. The simulations allow us to have a complete set of base configurations that could be used to perform configurational sampling and extract structural broadening effects; the analysis of this issue is left for future work.

All our simulations were done for systems in the gas phase. Note that the effect of a solvent may be crucial when comparing the computational results with experimental data. ${ }^{37,38}$ Furthermore, the sugar-phosphate backbone was neglected. This restriction should not be relevant in the energy range we are interested in $(3-7.5 \mathrm{eV})$, as the sugar and the phosphate contributions to the absorption spectra only start to be important above $7.0 \mathrm{eV}$.

b. Technical Details of the DFT Runs. The DFT structural optimization was carried out using the B3LYP/6-31++G(d,p) exchange-correlation functional for the isolated bases and GC assemblies, and the PW91 functional ${ }^{59}$ for the $\mathrm{AT}_{\mathrm{H}}$ pair. This latter functional was widely tested in guanine-rich systems, ${ }^{60-62}$ and yields a similar accuracy for $\mathrm{AT}_{\mathrm{H}}$ pairs and stacked pairs.

It is well-known that LDA may be grossly wrong in the description of H-bonds: that is why different gradient-corrected functionals were used to relax the geometries. However, we also know that, once the geometry is given, the LDA and GGA furnish very similar results for the excitations, therefore we stick to the LDA functional for simplicity. We are confident this is a reasonable approximation. Indeed, in the test mentioned above for guanine, different functionals do not yield significant differences as far as the optical excitations are concerned.

\section{Isolated Gas-Phase Nucleobases}

In this section we present the calculated absorption spectra for the five isolated nucleobases. Both the purines and the pyrimidines exist in nature in different tautomeric forms. We limited our calculations to the $9 \mathrm{H}$ keto form of guanine and to the $9 \mathrm{H}$ amino form of adenine. The latter tautomer is the one 


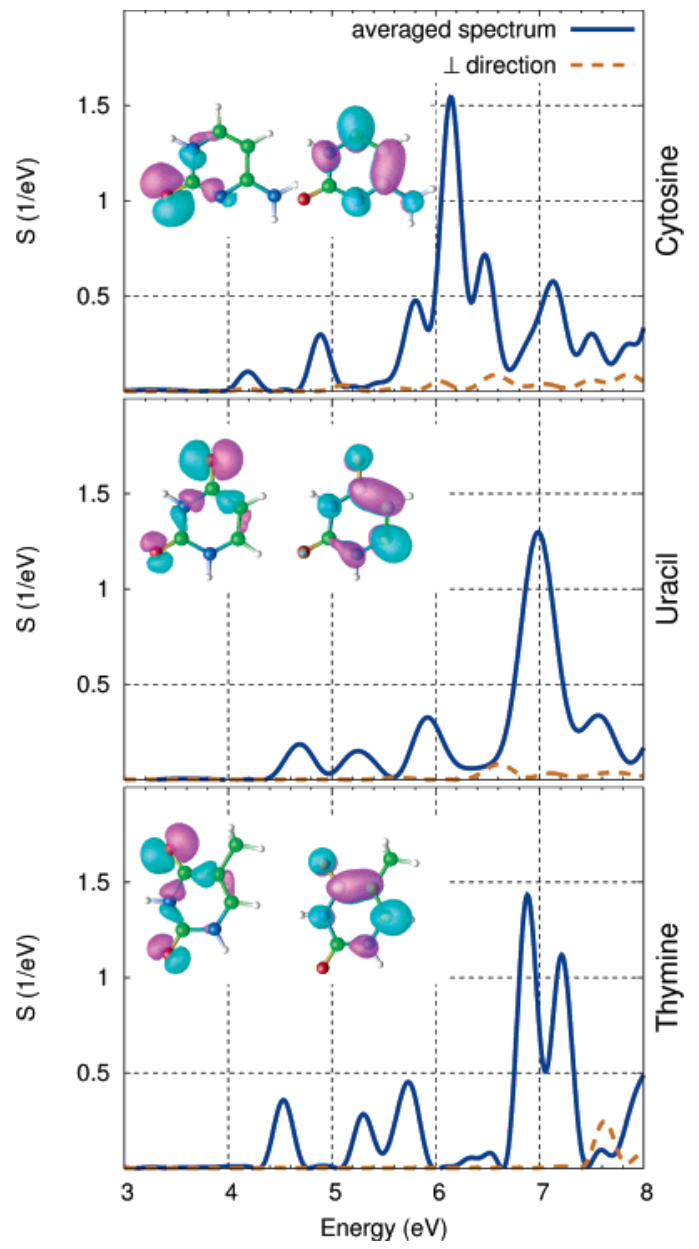

Figure 2. Photoabsorption cross section of isolated gas-phase pyrimidine nucleobases. The solid blue (dashed yellow) line is the signal averaged along the three real-space axes (projected onto the axis perpendicular to the base plane). Insets: HOMO (left) and LUMO (right) Kohn-Sham wave functions. The cyan (magenta) isosurfaces represent positive (negative) charge values. Different atoms are indicated with different colors: carbon (green), nitrogen (blue), oxygen (red), hydrogen (white).

TABLE 1: Vertical Excitations Energies (eV) Calculated for Cytosine, Compared with Averaged Experimental Values and Selected Computational Results ${ }^{a}$

\begin{tabular}{ccccc}
\hline exp. & TDDFT(a) & TDDFT(b) & CASPT2(c) & TDDFT(d) \\
\hline 4.6 & 4.18 & 4.10 & 4.39 & 4.65 \\
$5.0-5.3$ & 4.88 & 4.90 & 5.36 & 5.39 \\
$5.4-5.8$ & 5.80 & 5.92 & & 6.11 \\
$6.1-6.3$ & 6.14 & 6.39 & 6.16 & 6.32 \\
& 6.47 & 6.48 & 6.74 & 6.46 \\
$6.7-7.1$ & 6.88 & 6.88 & 7.61 & \\
& 7.13 & 7.16 & &
\end{tabular}

${ }^{a}$ The columns correspond to: (a) this work; (b) ref 25; (c) ref 30; (d) ref 26. The experimental absorption and CD values, along with the original references, are collected in ref 30.

present in DNA and RNA polymers and is therefore the most relevant regarding the adenine properties in nucleic acids.

3.1 Pyrimidines. a. Cytosine. The computed total and perpendicular photoabsorption cross sections of cytosine are shown in Figure 2. The energies of the spectral peaks are reported in Table 1, together with other selected sets of data that represent the state-of-the-art performance of experimental and computational techniques on cytosine.

For what concerns the transition energies, an overall glance at Table 1 reveals a rather good agreement of our results with
TABLE 2: Vertical Excitations Energies (eV) Calculated for Uracil, Compared with Average Experimental Values and Selected Calculations ${ }^{a}$

\begin{tabular}{ccll}
\hline exp. & TDDFT(a) & CASPT2(b) & TDDFT(c) \\
\hline $4.6-4.9$ & 4.69 & $4.54\left(n \pi^{*}\right)$ & $4.66\left(n \pi^{*}\right)$ \\
$4.9-5.2^{d}$ & 5.25 & 5.00 & 5.17 \\
$5.8-6.1$ & 5.92 & 5.82 & 5.89 \\
$6.3-6.6$ & 6.28 & 6.46 & 6.42 \\
$6.8-7.0$ & 6.98 & 7.00 & 6.81 \\
& 7.57 & &
\end{tabular}

${ }^{a}$ The columns correspond to: (a) this work; (b) ref 28; (c) ref 26; (d) this band was obtained in CD spectra and it was argued that it exhibits $n \pi^{*}$ character. The experimental absorption and CD values, along with the original references, are collected in ref 28 ; the second experimental band is taken from refs 67 and 68 .

those obtained in ref 25 , and a poorer matching with the outcome of B3LYP-TDDFT based on Gaussian $98,{ }^{26}$ and of CASPT2. None of the computational data is in perfect accord with experiment. Therefore, a selection of the best theoretical approach on the basis of the comparison between theory and experiment is hindered. Our agreement with experimental values is much more satisfactory in the high-energy than in the lowenergy regime.

If we turn our attention to the relative oscillator strengths between the various peaks, then the agreement with the data of ref 25 is not so perfect: we encounter differences in the distribution of oscillation strengths in the high-energy range of the spectrum. We will see later that these high energy discrepancies also appear in the spectra of the other nucleobases and can be traced to the limitations of the localized basis set used in ref 25 , and absent in the present work due to the use of a real-space grid.

Combining the time-domain spectra with the frequencydomain analysis, we can describe the spectral peaks in terms of transitions between electron states. Figure 1 shows an $n \pi^{*}$ transition at $4.3 \mathrm{eV}$ in the perpendicular spectrum, not clearly discernible in Figure 2 because of the low intensity of the perpendicular signal. This means that the lowest energy peak at $4.18 \mathrm{eV}$ in the averaged spectrum (Table 1), absent in the perpendicular polarization, must have a $\pi \pi^{*}$ character. The fact that the first optical excitation in cytosine is $\pi \pi^{*}$-like was predicted in all recent computational studies. The spacing between the lowest $\pi \pi^{*}$ excitation and the $n \pi^{*}$ transition computed by us is $0.12 \mathrm{eV}$ : this value is in good agreement with the results of B3LYP-TDDFT ${ }^{26}$ and CIS, ${ }^{63}$ whereas CASPT2 calculations ${ }^{30,64}$ predict a larger spacing between the two excitations. A smaller spacing was found in ref 65 with B3LYP-TDDFT.

b. Uracil and Thymine. The computed total and perpendicular photoabsorption cross sections of uracil and thymine are shown in Figure 2. The energies of the spectral peaks are reported in Tables 2 and 3 . The spectra of the two bases $U$ and $T$ are very similar, both in the number of peaks and spectral energies. A fairly good agreement is met with averaged experimental data, as well as with TDDFT ${ }^{25,26}$ and CASPT2 $2^{28}$ computational data.

All previous calculations, either in the TDDFT framework or with quantum chemistry methods, predict the lowest transition to have an $n \pi^{*}$ character in vacuo. We agree with this assignment for uracil (the perpendicular signal is not visible at $4.69 \mathrm{eV}$ in Figure 2 due to the weak intensity). In the case of thymine, we do not find any appreciable signal perpendicular to the base plane contributing to the peak at $4.54 \mathrm{eV}$. However, this is consistent with $\mathrm{TDDFT}^{26}$ and CASPT2 ${ }^{28}$ calculations, as they indeed report $n \pi^{*}$ transitions with extremely small oscillation strengths (1e-4-1e-6), unresolved by us. 
TABLE 3: Vertical Excitations Energies (eV) Calculated for Thymine, Compared with Average Experimental Values and Selected Calculations ${ }^{a}$

\begin{tabular}{lcclc}
\hline exp. & TDDFT(a) & TDDFT(b) & CASPT2(c) & TDDFT(d) \\
\hline $4.5-4.7$ & 4.54 & 4.45 & $4.39\left(n \pi^{*}\right)$ & 4.69 \\
$5.0-5.1$ & 5.30 & 5.24 & 4.88 & 4.96 \\
$5.8-6.0$ & 5.74 & 5.68 & 5.88 & 5.95 \\
$6.3-6.6$ & 6.36 & 6.38 & 6.10 & 6.19 \\
7.0 & 6.51 & & & 6.50 \\
& 6.88 & 6.86 & 7.13 & 6.86 \\
& 7.21 & 7.07 & & \\
& 7.59 & 7.28 & &
\end{tabular}

${ }^{a}$ The columns correspond to: (a) this work; (b) ref 25; (c) ref 28; (d) ref 26. The experimental absorption and $\mathrm{CD}$ values, along with the original references, are collected in ref 28; the experimental second band is taken from refs 67 and 68 .

The character of the band at $4.9-5.2 \mathrm{eV}$ for uracil and at 5.0-5.1 eV for thymine is not yet clear: the absorption spectra of uracil compounds ${ }^{66}$ and circular dichroism experiments ${ }^{67}$ indicate that it is due to a $\pi \pi^{*}$ transition. However, such $\pi \pi^{*}$ character has not been confirmed by magnetic circular dichroism ${ }^{69}$ and polarized absorption spectra experiments. ${ }^{70-73}$ Lorentzon and co-workers, by performing calculations at the CASPT2 level, ${ }^{28}$ suggest that this band has a $n \pi^{*}$ origin: they arrived at this conclusion by correcting their computed values with a 0.5 $\mathrm{eV}$ blue-shift due to the solvent. A similar conclusion was reported for uracil by Shukla and co-workers, ${ }^{39}$ who performed CIS calculations taking the solvent into account with a polarizable continuum model. However, more recently, Shukla and Leszczynski using B3LYP-TDDFT ${ }^{26}$ reported only a $\pi \pi^{*}$ transition at $5.17 \mathrm{eV}$ for uracil and at 4.96 for thymine in the energy range under consideration. In our work, we do not detect any absorption in the direction perpendicular to the base-plane in this energy range, whereas peaks at $5.24 \mathrm{eV}$ for $\mathrm{T}$ and at $5.25 \mathrm{eV}$ for $\mathrm{U}$ are found, with considerable oscillator strength induced by light polarized in the plane of the molecule. Therefore, our results indicate that the second band most likely has a $\pi \pi^{*}$ character.

3.2 Purines. a. Adenine. The computed total and perpendicular photoabsorption cross sections of $9 \mathrm{H}$-adenine are shown in Figure 3. The spectral peaks are summarized in Table 4 and compared with other available theoretical and experimental data. An excellent agreement with other LDA-TDDFT computed values $^{25}$ is observed, regarding both the peak energies and the relative oscillation strengths. Slight discrepancies occur only in the high energy range: these are most likely due to differences in the technical details adopted in the calculations (e.g., the basis sets).

Looking at the spectrum in the direction perpendicular to the base plane, we can distinguish four prevalently $n \pi^{*}$ transitions in the energy range 4.10-4.79 eV, which are hidden in Figure 3 because of their tiny oscillator strengths. We find that the lowest excitation has mainly $n \pi *$ character and is very close in energy to the first $\pi \pi^{*}$ transition (forming the peak at $4.51 \mathrm{eV}$ ). This result is in agreement with resonant two-photon ionization and laser-induced fluorescence spectroscopy of jet-cooled adenine ${ }^{74}$ provided that the adiabatic transition energies follow the same trends as the calculated vertical ones. Our findings also agree qualitatively with frequency-domain B3LYP-TDDFT $^{26}$ and CIPSI ${ }^{37,75}$ calculations, whereas CASPT2 [29] and CIS $^{38,76,77}$ yield the reverse order. Given the small energy difference between the two transitions, we can conclude that the overall qualitative agreement is satisfactory.

Proceeding to higher energies, we find the second $\pi \pi^{*}$ peak $0.37 \mathrm{eV}$ after the first one. Experiments indicate ${ }^{76-79}$ that the

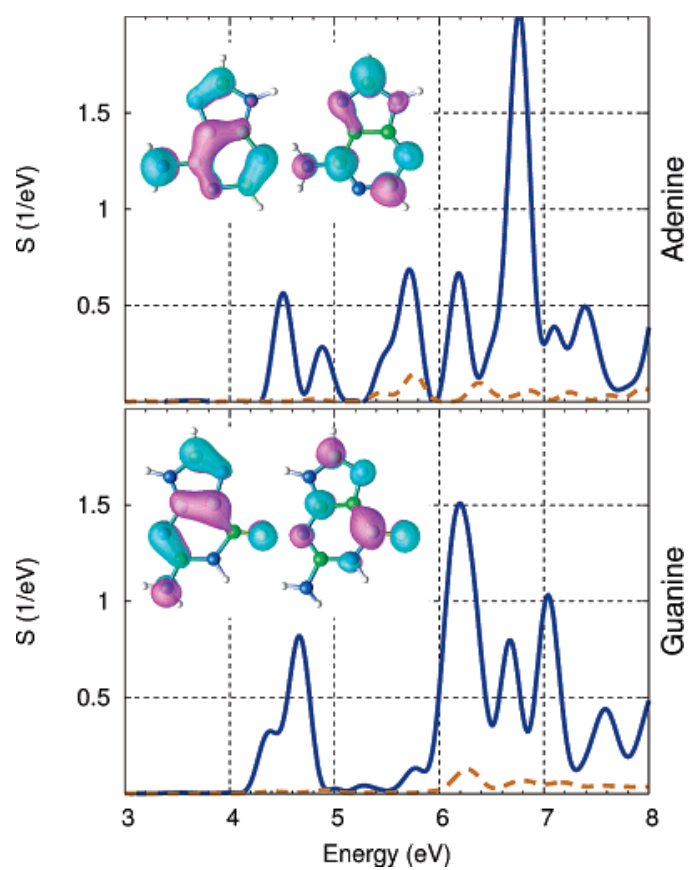

Figure 3. Same as Figure 2 for adenine and guanine.

TABLE 4: Vertical Excitations Energies (eV) Calculated for Adenine, Compared with Averaged Experimental Values and Selected Calculations

\begin{tabular}{lcccc}
\hline \multicolumn{1}{c}{ Exp. } & TDDFT (a) & TDDFT(b) & CASPT2(c) & TDDFT(d) \\
\hline 4.59 & 4.51 & 4.51 & 5.13 & 4.94 \\
$4.8-4.9$ & 4.88 & 4.95 & 5.20 & 5.21 \\
5.38 & 5.49 & 5.58 & & \\
$5.7-6.1$ & 5.72 & 5.79 & 6.24 & 5.93 \\
6.26 & 6.19 & 6.28 & 6.72 & 6.12 \\
& 6.49 & 6.63 & & 6.16 \\
6.81 & 6.76 & 6.92 & 6.99 & \\
& 7.09 & 7.47 & & \\
7.73 & 7.39 & 7.81 & 7.57 &
\end{tabular}

The columns correspond to: (a) this work; (b) Ref. 25; (c) Ref. 29; (d) Ref. 26. The experimental absorption and CD values, along with the original references, are collected in Ref. 29.

low energy portion of the adenine photoabsorption spectrum consists of two closely spaced $\pi \pi^{*}$ transitions. Holmén and coworkers showed by linear dichroism that those two peaks are separated by $0.26 \mathrm{eV} .^{76}$ This value is in reasonable agreement with our results and with other TDDFT calculations, ${ }^{25,37}$ while different techniques predict a closer energy spacing. On the other hand, measured spectra indicate that the lowest energy transition has a smaller oscillator strength than the subsequent one: such an evidence is consistent with the CASPT2 and CIPSI results, whereas TDDFT simulations yield an inverse ordering of oscillator strengths in the first two peaks ${ }^{25}$ (see Figure 3).

Concerning other $n \pi^{*}$ transitions, there is experimental evidence by $\operatorname{circular}^{68,80}$ and linear ${ }^{76}$ dichroism of a signal around $5.4 \mathrm{eV}$. Our calculation is also able to reveal a signal in this range. However, the agreement with the experiment for the $n \pi^{*}$ transitions should be considered with caution, because of their high sensitivity to the effect of the solvent (neglected in our calculations) and exchange-correlation functional.

$b$. Guanine. To conclude the presentation of the results for the isolated DNA bases, we show in Figure 3 the computed total and perpendicular photoabsorption cross sections of $9 \mathrm{H}-$ guanine. The spectral peaks are summarized in Table 5 and compared with other available data. The excitation energies are in good agreement with the averaged experimental data and with 
TABLE 5: Vertical Excitation Energies (eV) Calculated for Guanine, Compared with Averaged Experimental Values and Selected Calculations ${ }^{a}$

\begin{tabular}{ccccc}
\hline Exp. & TDDFT $^{\text {a }}$ & TDDFT $^{\mathrm{b}}$ & CASPT2 $^{\mathrm{c}}$ & TDDFT $^{\mathrm{d}}$ \\
\hline $4.4-4.5$ & 4.40 & 4.46 & 4.76 & 4.85 \\
$4.9-5.0$ & 4.66 & 4.71 & 5.09 & 5.11 \\
& 5.01 & 5.04 & & \\
$5.7-5.8$ & 5.28 & & & \\
$6.1-6.3$ & 5.76 & 5.64 & 5.96 & 5.59 \\
$6.6-6.7$ & 6.22 & 6.23 & 6.55 & 5.83 \\
& 6.67 & 6.53 & 6.65 & \\
& & 6.82 & 6.66 & \\
& 7.04 & 6.93 & & \\
& 7.58 & 7.26 & &
\end{tabular}

${ }^{a}$ The columns correspond to (a) this work; (b) ref 25; (c) ref 29; (d) ref 26. The experimental absorption and $C D$ values, along with the original references, are collected in ref 29.

the results of ref 25. Small differences are encountered in the high energy region of the spectrum in the relative oscillator strengths of the peaks at $6.22 \mathrm{eV}$ and $6.67 \mathrm{eV}$, while the low energy region is in perfect agreement. Again, we attribute the discrepancies at higher energies to the different basis sets used in ref 25 and in our work. In the region from 5 to $6 \mathrm{eV}$ we also find very weak peaks that are not observed experimentally. Regarding the out-of-plane spectrum, we find one peak at 4.47 $\mathrm{eV}$ that has a purely $n \pi^{*}$ character. Thus, the first $n \pi^{*}$ transition has a higher energy than the first $\pi \pi^{*}$ transition found at 4.40 $\mathrm{eV}$ (see Table 5). This attribution is consistent with previous CASPT2 [29], CIS, ${ }^{39}$ and B3LYP-TDDFT ${ }^{81}$ calculations.

3.3 General Comments on the Results for the Isolated GasPhase Nucleobases. The results of our time-dependent calculations ${ }^{41}$ of the excitation spectra of DNA bases and uracil in the gas phase show a satisfactory agreement with the experimental data and with other computational approaches, especially for the most intense peak above $6 \mathrm{eV}$. In particular, good agreement is found with recent LDA-TDDFT calculations using a localized-basis set. ${ }^{25}$ Minor differences are found regarding the distribution of the oscillator strengths in the high energy range of the spectra. Such discrepancies can be ascribed to the different basis sets: a uniform grid in real space in this work and a set of localized atomic orbitals in ref 25 .

Regarding the more problematic $n \pi^{*}$ transitions, we find that the weak perpendicular signal is sometimes hidden below the in-plane signal. In the cases where the $n \pi^{*}$ transitions can be detected, we find the correct relative ordering between $n \pi^{*}$ and $\pi \pi^{*}$ peaks, as compared with other TDDFT calculations performed in the frequency domain. Another interesting trend is the relative ordering among different nucleobases for what concerns the first $\pi \pi^{*}$ transition. For this feature, supersonic jet experiments report $\mathrm{C}<\mathrm{G}<\mathrm{A} .{ }^{74,82,83} \mathrm{We}$ indeed find that ordering in our first-principles calculations, i.e., the first excitation at $4.18 \mathrm{eV}$ for cytosine, $4.40 \mathrm{eV}$ for guanine and at $4.52 \mathrm{eV}$ for adenine. Last, we note that our calculations give the expected character of the Kohn-Sham orbitals of the different bases (see insets in Figures 2 and 3): the LUMO has $\pi$ character for all the nucleobases, whereas the HOMO is $\pi$-like for the purines and $\sigma$-like for the pyrimidines, with a high charge density around the oxygen atoms in all cases.

\section{Watson-Crick Pairs $\mathrm{GC}_{\mathrm{H}}$ and $\mathrm{AT}_{\mathrm{H}}$}

We now turn the discussion to the standard assembly of DNA bases, namely the Watson-Crick pairing. Figure 4 shows the calculated TDDFT spectra of $\mathrm{H}$-bonded $\mathrm{GC}_{\mathrm{H}}$ and $\mathrm{AT}_{\mathrm{H}}$ pairs (left) and the spectra resolved in the direction perpendicular to the basal plane (right). The sum of the photoabsorption cross sections from the isolated component nucleobases is also shown by the dashed lines (indicated by $\mathrm{G}+\mathrm{C}$ and $\mathrm{A}+\mathrm{T}$ ), to reveal the effects induced by $\mathrm{H}$-bonding in the Watson-Crick pairs. The peaks observed in the spectra are also listed in Table 6 for a more detailed analysis. Figure 5 illustrates the isosurface plots of the HOMO and LUMO, which are the single-particle orbitals most important for the optical transitions in the energy range discussed here. In both pairs, the HOMO is localized at the purine and the LUMO at the pyrimidine. The HOMO-LUMO gaps are 1.93 and $3.07 \mathrm{eV}$ for the $\mathrm{GC}_{\mathrm{H}}$ and $\mathrm{AT}_{\mathrm{H}}$ pairs, respectively.

We focus our analysis on the most evident features induced by H-bond base pairing, which are: (i) a small shift found for the lowest frequency peaks; (ii) the hypochromicity (intensity decrease) at high frequencies; (iii) an overall blue-shift of the spectrum for light polarized perpendicular to the pair.

The relaxed structure of the $\mathrm{GC}_{\mathrm{H}}$ pair is practically planar. The shape of the total photoabsorption spectrum of the hydrogen-bonded $\mathrm{GC}_{\mathrm{H}}$ pair remains rather similar to the linear superposition of the isolated bases (cf. the top left panel of Figure 4). The first $\pi \pi^{*}$ peak at $4.29 \mathrm{eV}$ is composed of the first excitation of cytosine and the first excitation of guanine. We note a slight red shift of this peak with respect to the first peak in the red curve: this effect is the combination of a blue shift by about $0.1 \mathrm{eV}$ of the first cytosine peak and a comparable red shift of the first guanine peak. Such finding is in qualitative agreement with a CASPT2 calculation by Sobolewski and Domcke $^{33}$ and by a calculation performed by Wesolowski ${ }^{34}$ by embedding methods.

For the second $\pi \pi^{*}$ transition at $4.72 \mathrm{eV}$ the situation is reversed. The peak is blue-shifted with respect to the superposition of isolated $\mathrm{G}$ and $\mathrm{C}$. This shift results from a blue shift of the second guanine peak and a red shift of the second cytosine peak. In the high-energy spectral range, we remark that the effect of the H-bonding pairing does not change the position of the brightest peak at $6.17 \mathrm{eV}$, which has both $\mathrm{G}$ and $\mathrm{C}$ components. However, the intensity of this peak is depressed (hypochromism) by about $30 \%$ with respect to the free monomers. Above this energy the spectrum results much more changed.

Looking at the direction perpendicular to the plane of the $\mathrm{GC}_{\mathrm{H}}$ pair (top right panel), we observe an overall blue shift of the spectrum. In particular, the first peak is shifted upward by $0.48 \mathrm{eV}$. This behavior reflects the well-known uplifting of $n \pi^{*}$ excitation energies in hydrogen-bonding environments, already pointed out by other authors, ${ }^{88,89}$ and appears naturally from our first-principles simulation.

For the $\mathrm{AT}_{\mathrm{H}}$ pair, Table 6 highlights an overall good agreement with a recent LDA-TDDFT calculation. ${ }^{25}$ Small differences can be reasonably imputed to the different structure obtained upon relaxation. We find an almost planar geometry, in agreement with Shukla and Leszczynski, ${ }^{32}$ while Tsolakidis and Kaxiras ${ }^{25}$ find a propeller structure.

As in the case of the $\mathrm{GC}_{\mathrm{H}}$ pair, the overall spectrum of $\mathrm{AT}_{\mathrm{H}}$ is quite similar to the linear combination of the spectra of the isolated $\mathrm{A}$ and $\mathrm{T}$ bases. A small blue shift, by $\sim 0.1 \mathrm{eV}$, is detected in the low-energy range (roughly below $6 \mathrm{eV}$ ) with respect to the superposition of isolated bases. In the high-energy part of the spectrum, we observe an enhancement of the strength with respect to isolated adenine and thymine for the peak located at $6.78 \mathrm{eV}$ and a reduction for the peak at $7.18 \mathrm{eV}$ present in the isolated thymine spectrum. Hence, for the most intense peak at $6.78 \mathrm{eV}$, the effect of $\mathrm{H}$-bonding is contrary to the hypochromicity described above for the $\mathrm{GC}_{\mathrm{H}}$ pair. 

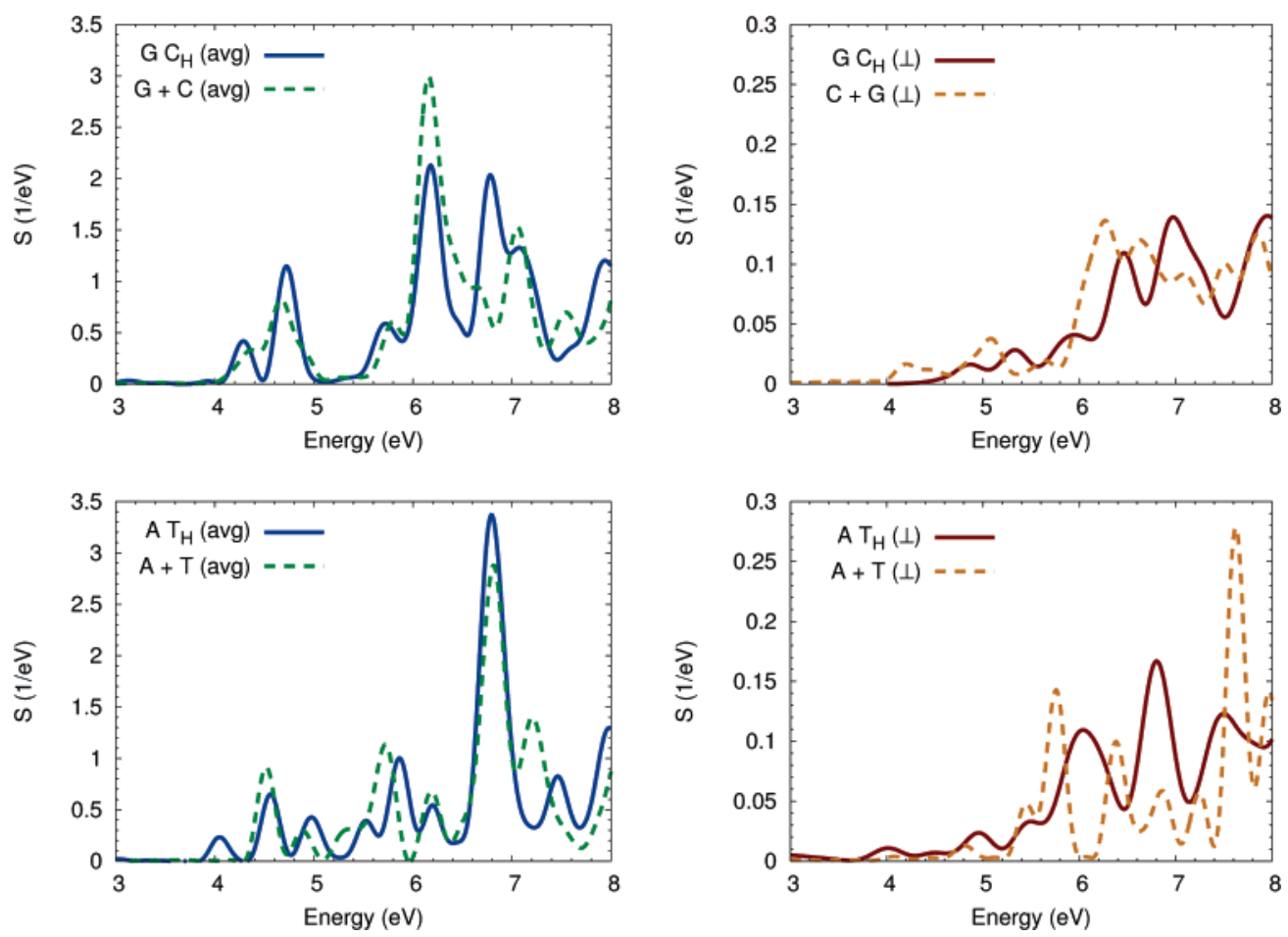

Figure 4. Photoabsorption cross section of the $\mathrm{GC}_{\mathrm{H}}$ and $\mathrm{AT}_{\mathrm{H}}$ base pairs averaged (solid blue, left) and in the direction perpendicular to the base plane (solid red, right). The linear combinations of the spectra of the isolated purine and corresponding pyrimdine $(\mathrm{G}+\mathrm{C}$ and $\mathrm{A}+\mathrm{T}$; dashed lines) are also shown for comparison.

TABLE 6: Vertical Excitation Energies (eV) for Watson-Crick GCH and ATH Pairs, Compared to Other Calculations $^{a}$

\begin{tabular}{cccccc}
\hline & \multicolumn{2}{c}{$\mathrm{GC}$} & & \multicolumn{2}{c}{$\mathrm{AT}$} \\
\cline { 2 - 3 } \cline { 5 - 6 } TDDFT $^{(\mathrm{a})}$ & TDDFT $^{(\mathrm{b})}$ & CASPT2 $^{(\mathrm{c})}$ & & TDDFT $^{(\mathrm{a})}$ & TDDFT $^{(\mathrm{b})}$ \\
\hline 2.44 & 2.37 & & & \\
3.14 & 3.53 & & 4.05 & 4.05 \\
3.94 & & & & 4.26 \\
4.29 & 4.21 & 4.35 & & 4.56 & 4.40 \\
4.72 & 4.75 & 4.67 & & 4.80 \\
& & $4.75^{(\mathrm{d})}$ & & 5.98 \\
5.38 & 5.52 & & & 5.45 \\
5.72 & 5.69 & & 5.53 & 5.60 \\
& 5.84 & & 5.87 & 5.76 \\
6.17 & 6.07 & & 6.20 & 6.24 \\
6.46 & 6.33 & & & 6.48 \\
6.78 & 6.47 & & 6.78 & 6.73 \\
7.07 & 6.68 & & 7.80 & 6.97 \\
7.53 & 7.83 & & &
\end{tabular}

${ }^{a}$ The columns correspond to (a) this work; (b) ref 25; (c) ref 33 (cf. also the results of Shukla and Leszczynski ${ }^{32}$ ); (d) Charge-transfer transition.

In the out-of-plane spectrum (bottom right panel of Figure 4), as for the $\mathrm{GC}_{\mathrm{H}}$ pair, we observe that the effect of hydrogen bonding is an overall blue shift. In particular, the first peak (that has an $n \pi^{*}$ character) is shifted by $0.4 \mathrm{eV}$; the second and fourth peaks are upward shifted by $0.2 \mathrm{eV}$ and $0.3 \mathrm{eV}$ respectively; the energy of the third peak is practically unchanged.

In addition to the above features that originate from the individual bases that enter each pair, new features appear in the spectra because the purine and the pyrimidine coexist in the Watson-Crick arrangement. This coexistence changes the nature of the frontier orbitals: the HOMO is purine-localized, the LUMO is pyrimidine-localized, and the value of the HOMO-LUMO gap is smaller than in the isolated bases.

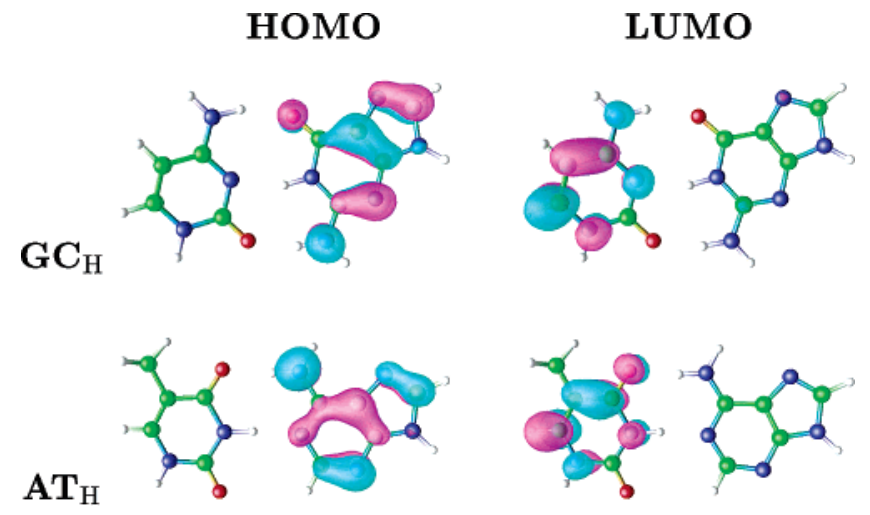

Figure 5. HOMO and LUMO Kohn-Sham wave functions of the $\mathrm{GC}_{\mathrm{H}}$ and $\mathrm{AT}_{\mathrm{H}}$ pairs.

Consequently, peaks at lower energies emerge in the spectra. They are reported in Table 6, but the corresponding energy range is not shown in the figures because the tiny oscillator strengths make them undiscernible from the more intense peaks. We also note that the extremely small intensity makes the quantitative assignment of these features less reliable than the others already discussed. In the case of $\mathrm{GC}_{\mathrm{H}}$, we find excitation energies at 2.44, 3.14, and $3.94 \mathrm{eV}$, with oscillator strengths of $0.001,0.008$, and 0.003 . These oscillator strengths are two orders of magnitude smaller than the peak at $4.29 \mathrm{eV}$ shown in the upper panel of Figure 4. For the $\mathrm{AT}_{\mathrm{H}}$ pair, the lowest energy peak at $4.05 \mathrm{eV}$ has an oscillator strength of 0.048 . This transition has a small component also in the absorption for light polarized perpendicular to the base plane (see bottom right panel in Figure 4). Its origin can be likely ascribed to charge transfer states, that have received particular attention from both experimental and theoretical viewpoints. ${ }^{84}$ However, we warn the reader that the identification of charge-transfer transitions is to be taken with care at the current level of TDDFT when a local or gradientcorrected exchange-correlation functional is used. ${ }^{85-87}$ 

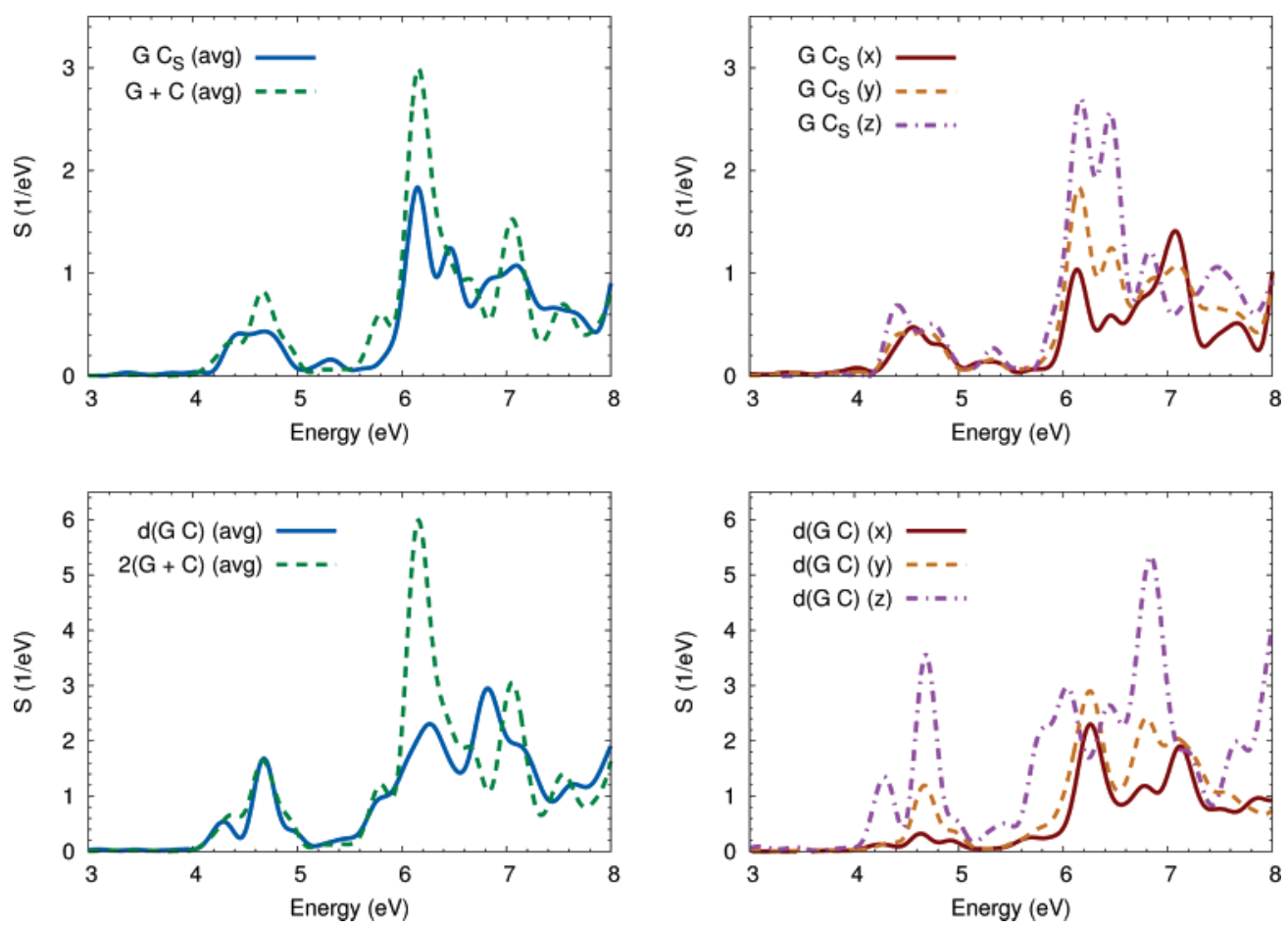

Figure 6. Photoabsorption cross section of the $\mathrm{GC}_{\mathrm{H}}$ stacked dimer (up) and of the $\mathrm{d}(\mathrm{GC})$ quartet (down). In each panel, the linear combination of the spectra of the two (up) and four (down) constituent bases is also shown. In the right panels the spectra resolved in the three spatial directions are shown.

HOMO
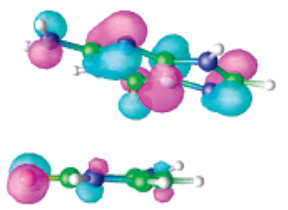

$\pi$ stacked bases

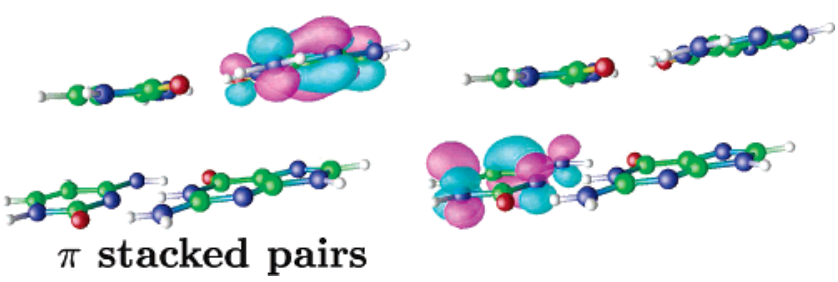

Figure 7. Isosurface plots of the HOMO and LUMO Kohn-Sham wave functions of the $\mathrm{GC}_{\mathrm{s}} \pi$-stacked dimer and of the $\mathrm{d}(\mathrm{GC}) \pi$-stacked quartet.

\section{Stacked $G_{\mathrm{s}}$ and $\mathrm{D}(\mathrm{GC})$ Structures}

With the results reported in the previous section for the $\mathrm{GC}_{\mathrm{H}}$ pair, we can understand what happens in the optical absorption when a guanine and a cytosine dimerize through hydrogen bonding. Here, the $\mathrm{GC}_{\mathrm{s}}$ system is selected to check which effects arise when the same two bases dimerize in a different form, namely by stacking.

Figure 6 reports the averaged and direction-resolved spectra of the stacked $\mathrm{GC}_{\mathrm{s}}$ dimer. The comparison between Figures 6 and 4 aims at disclosing the relative role of hydrogen bonding and $\pi$ stacking in the optical response of nucleobase complexes. We also plot in Figure 6 the linear combination of the spectra of the isolated constituent bases. Figure 7 (top) illustrates the Kohn-Sham highest occupied and lowest unoccupied orbitals of the system. It is important to note that both the HOMO and the LUMO have charge density distributed around both the purine and the pyrimidine, at odds with the $\mathrm{H}$-bonded $\mathrm{GC}_{\mathrm{H}}$ pair (compare Figure 5, top) where the HOMO is centered at the purine and the LUMO at the pyrimidine.

As in the case of hydrogen bonding, the shape of the spectrum is not strongly altered but important differences are encountered in the oscillator strengths. Looking at the low-energy peaks, we see that the excitations due to the cytosine moiety are slightly blue-shifted (as for the $\mathrm{GC}_{\mathrm{H}}$ pair), while the guanine excitations appear unaffected. The resulting spectrum below $5 \mathrm{eV}$ is made of two equally intense closely spaced peaks at $4.45 \mathrm{eV}$ and 4.68 $\mathrm{eV}$, whereas in the $\mathrm{H}$-bonded $\mathrm{GC}_{\mathrm{H}}$ structure we observe two well separated peaks spaced by $0.4 \mathrm{eV}$ and with different intensities. Hypochromicity is also observed in this low-energy range, whereas for the $\mathrm{GC}_{\mathrm{H}}$ pair hypochromicity occurs only in the high-energy range.

In the range between 5 and $6 \mathrm{eV}$, we find an enhancement of the weak peak at $5.31 \mathrm{eV}$ and a depression of the peak at $5.78 \mathrm{eV}$, which is present both in the isolated bases (guanine and cytosine) and in the H-bonded pair. In the high-energy spectral range, the dominant effect is the $39 \%$ hypochromicity for the main peak located at $6.15 \mathrm{eV}$ : hypochromicity from $\pi$-stacking is thus enhanced with respect to hypochromicity from hydrogen bonding (30\%). Above the strongest peak, we find other less intense peaks at $6.47 \mathrm{eV}, 6.87 \mathrm{eV}$, and $7.10 \mathrm{eV}$. Instead, in the $\mathrm{H}$-bonded $\mathrm{GC}_{\mathrm{H}}$ pair, the high-energy range contains a peak at $6.78 \mathrm{eV}$ with the same intensity as the peak at $6.17 \mathrm{eV}$, and then only an additional weaker one.

The calculation of the $\mathrm{GC}_{\mathrm{s}}$ has been repeated for a geometry obtained by a slightly different relaxation scheme, within the DFT-PW91 framework. ${ }^{61,62}$ This additional $\mathrm{GC}_{\mathrm{s}}$ geometry was considered just for a gross check of structural deformations induced by computational details. The starting configuration was fixed by taking guanine and cytosine from a previous DFT study of isolated bases ${ }^{8}$ and putting them parallel to each other at an initial distance of $3.4 \AA$. The major difference between the two 

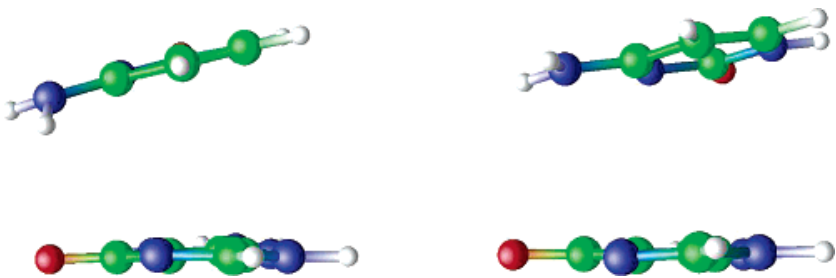

Figure 8. Two different conformations obtained for the $\pi$-stacked $\mathrm{GC}_{\mathrm{s}}$ pair obtained by different structural relaxation schemes.

$\mathrm{GC}_{\mathrm{s}}$ structures (Figure 8) is the tilt angle, which is smaller in the PW91 relaxation. The two spectra are quite similar in both shape and intensity: minor differences are observed only in the first peak, which is shifted upward by $0.15 \mathrm{eV}$ in the structure shown in the right panel of Figure 8.

The combined effects of stacking and H-bonding on the absorption spectra are simultaneously present in Figure 6 (lower panel), which corresponds to two stacked $\mathrm{GC}_{\mathrm{H}}$ pairs with the G's on the same strand of the parent polymer. ${ }^{55}$ The shape of the spectrum appears very similar to that of a single $\mathrm{GC}_{\mathrm{H}}$ pair (Figure 4, top left), but the intensity of all peaks is reduced. This decrease is a typical qualitative effect of the stacking arrangement, although the molecular details fix the quantitative aspects (we described the stacking between guanine and cytosine above, whereas we are now focusing on the stacking between two guanines). In fact, in the low-energy range we find again very slight shifts for the first two peaks, downward for the first and upward for the second, as in Figure 4 (top left). However, the intensity of the second peak is equal to that obtained from the $\mathrm{G}+\mathrm{C}$ combination, whereas in the single $\mathrm{GC}_{\mathrm{H}}$ pair it was higher. This is due to a depression of the second peak (guanine) induced by stacking.

In the high-energy range, the hypochromicity due to both the $\pi$-stacking and the H-bonding couplings is confirmed. For the main peak located at $6.25 \mathrm{eV}$ we find an intensity reduction by $62 \%$ with respect to the free monomers. The two kinds of base couplings seem to act separately and independently, in the sense that one does not affect the other: such separation was already reported concerning the electronic structure. ${ }^{8}$

Summarizing this section, we have shown that stacked and $\mathrm{H}$-bonded GC pairs present slight differences in the absorption spectra, both in the low-energy and high-energy ranges. Hypochromicity has been found in both configurations and is largest for the stacked pairs. Hypochromicity is very useful because this intensity change can be used to follow the melting of the secondary structure of nucleic acids when varying the temperature or environmental parameters.

\section{Summary and Perspectives}

In this work we presented a complete study of the optical absorption spectra of the five isolated gas-phase nucleobases and their assemblies: simple Watson-Crick pairs, simple $\pi$-stacks of two bases and more complex $\pi$-stacks of WatsonCrick GC pairs. These calculations of isolated simple nucleobase-assemblies are a remarkable playground to prove the reliability of TDDFT for DNA-based materials. Remarkably, for the first time the optical properties were computed by an ab initio method for a helical conformation of two stacked GC pairs, where $\mathrm{H}$-bonding and $\pi$-stacking effects are active simultaneously and can be distinguished.

The results can be summarized as follows. For the isolated bases we get spectra in good agreement with previous theoretical works and (qualitatively) with experiments. We reproduce the proper ordering of the $\pi \pi^{*}$ excitations, namely the excitation energy increases in going from $\mathrm{C}$ to $\mathrm{G}$ to $\mathrm{A}$. Moreover, the
LUMO state has always a $\pi$-like character whereas the HOMO is $\pi$-like for the purines and $\sigma$-like for the pyrimidines. As concerns the base assemblies (Watson-Crick H-bond pairs and stacked configuration), we obtain that the shape of the spectrum is not much altered by the $\pi$-stacking or $\mathrm{H}$-bond interactions. However, we always get hypochromicity in the high energy range of the spectrum. The hypochromicity induced by $\pi$-stacking is larger than that induced by $\mathrm{H}$-bonding. For light polarized perpendicular to the bases we get a blue shift of the spectra compared to the spectra of the isolated bases. In the stacked case, the HOMO and LUMO states are distributed both on the purine and pyrimidine bases, whereas in the H-bonded configuration the HOMO is in the purine and the LUMO in the pyrimidine (charge-transfer-type excitation). When combining both $\mathrm{H}$-bonding and $\pi$-stacking, the two effects add independently and the hypochromicity in the UV is enhanced.

At this stage it is relevant to note that all our calculations are in the gas phase, which means that solvent and environmental effects are not taken into account. This hinders a direct comparison with experimental data, as most of the available results correspond to nucleobase assemblies in solution. However, several experimental and theoretical studies (including simplified models of the solvent) showed that the $\pi \pi^{*}$ transitions are only slightly affected by the presence of the solvent: ${ }^{32,37,38}$ precisely, they are insensitive to the polarity of the solvent, but may undergo red shifts. On the contrary, the "dark" $n \pi^{*}$ transitions turn out to be significantly sensitive to the polarity of the solvent. ${ }^{29,32,37-40}$ As shown in Figures 2 and 3, the absorption spectra of the DNA bases are extremely anisotropic, due to their quasi-planar structure. Consequently, nearly all the oscillator strength is concentrated on the $\pi \pi^{*}$ transitions (orders of magnitude more intense than the $n \pi^{*}$ transitions). Those transitions are excited only for light polarized in the base plane. Therefore, these results can be used to discriminate (albeit qualitatively) which features are mostly limited to the gas phase (those polarized out-of-plane) and which ones can be considered intrinsic to the DNA complex. ${ }^{90}$ Furthermore, the role of chargetransfer states needs to be analyzed more in detail in order to understand their impact in the excited state dynamics of DNAbased complexes (note that those states are very likely to be dark, or with very low oscillator strength for light-induced electronic excitations).

In addition to optical absorption, there is another optical technique that is widely used for the characterization of chiral biomolecules; circular dichroism. The computational method used in the present work allows for a straightforward calculation of the rotatory power or circular dichroism spectra, keeping the simplicity of the time-propagation scheme, i.e, without the need for empty states (see ref 44 for the details). The implementation and computation of circular dichroism spectra by TDDFT is in progress and will be the topic of a self-standing investigation ${ }^{91}$ that should allow the identification of helical fingerprint in the optical characteristics and more direct interpretation of standard post-synthesis experimental data.

Acknowledgment. The authors thank X. Lopez, E. Sansebastian, A. Calzolari, A. Garbesi, and E. Molinari for fruitful discussions. Funding was provided by the EC through grant IST2001-38951, by the EC 6th Framework Network of Excellence NANOQUANTA (NMP4-CT-2004-500198), by the EC 5th Framework Marie Curie Network EXC!TING, by Spanish MEC, and by the INFM through the Parallel Computing Committee. A.R. acknowledges the Humboldt Foundation under the Bessel research award (2005). We thank the SGI of the UPV and DIPC and the Barcelona supercomputer center for providing computer time. 


\section{References and Notes}

(1) Daniels, M. In Photochemistry and Photobiology of Nucleic Acids: Wang S. Y. Ed; Academic Press: New York, 1976; p 23.

(2) Hernández, C. E. C.; Cohen, B.; Hare, P. M.; Kohler, B. Chem. Rev. 2004, 104, 1977

(3) Ruzsicska, B. P.; Lemaire, D. G. E. In CRC Handbook of Photochemistry and Photobiology; Horspoll, W. M.; Lemaire, D. G. E., Eds; CRC Press: Boca Raton, Fl, 1995; p 1289.

(4) Porath, D.; Cuniberti, G.; Felice, R. D. In Topics in Current Chemistry; Schuster G. Ed.; Springer-Verlag: Heideleberg, 2004; p 183.

(5) Di Ventra, M.; Zwolak, M. In Encycolpedia of Nanoscience and Nanotechnology and Handbook of Nanostructured Biomaterials and their application in Nanotechnology; Nalwa H. S., Ed.; APS: College Park, MD, 2004; p 475.

(6) Endres, R. G.; Cox, D.; Singh, R. Rev. Mod. Phys. 2004, 76, 195.

(7) Coehn, H.; Nogues, C.; Naaman, R.; Porath, D. Proc. Natl. Acad. Sci. U.S.A. 2005, 102, 11589

(8) Di Felice, R.; Calzolari, A.; Molinari, E.; Garbesi, A. Phys. Rev. B 2002, 65, 045104 .

(9) Di Felice, R.; Calzolari, A. In Modern methods for theoretical physical chemestry of biopolymers; Starikov, E. B.; Tanaka, S.; Lewis J. P., Eds; Elsevier: Amsterdam, 2005.

(10) Kraemer, K. H. Proc. Natl. Acad. Sci. U.S.A. 1997, 94, 11.

(11) Odom, D. T.; Barton, J. K. Biochemistry 2001, 40, 8727.

(12) Hernández, C. E. C.; Kohler, B.; Cohen, B. Nature 2005, 436, 1141

(13) Watson, J. H.; Crick, F. H. Nature 1953, 171, 737.

(14) Hall, D. B.; Holmlin, R. E.; Barton, J. K. Nature 1996, 3841, 731.

(15) Runge, E.; Gross, E. K. U. Phys. Rev. Lett. 1984, 52, 997.

(16) Gross, E. K. U.; Dobson, J. F.; Petersilka, M. Density functional theory II, Topics in Current Chemistry; Nalewajski, R. F., Ed; SpringerVerlag: Berlin, 1996.

(17) Marques, M. A. L.; Gross, E. K. U. In A primer in Density Functional Theory; Fiolhais C., Nogueira F., Marques M. A. L., Eds; Springer: Berlin, 2003; p 144

(18) Onida, G.; Reining, L.; Rubio, A. Rev. Mod. Phys. 2002, 74, 601.

(19) Furche, F.; Burke, K. Annu. Rev. Comput. Chem. 2005, 1, 19.

(20) Marques, M. A. L.; Lopez, X.; Varsano, D.; Castro, A.; Rubio, A. Phys. Rev. Lett. 2003, 90, 258101.

(21) Lopez, X.; Marques, M. A. L.; Castro, A.; Rubio, A. J. Am. Chem. Soc. 2005, 127, 12329.

(22) Sundholm, D. Phys. Chem. Chem. Phys. 2000, 2, 2275.

(23) Sundholm, D. Phys. Chem. Chem. Phys. 2003, 5, 4265.

(24) Neiss, C.; Saalfrank, P.; Parac, M.; Grimme, S. J. Phys. Chem. A 2003, 107, 140

(25) Tsolakidis, A.; Kaxiras, E. J. Phys. Chem. A 2005, 109, 2373.

(26) Shukla, M. K.; Leszczynski, J. J. Comput. Chem. 2004, 25, 768.

(27) von Lilienfeld, O. A.; Tavernelli, I.; Rothlisberger, U.; Sebastiani, D. Phys. Rev. Lett. 2004, 93, 153004.

(28) Lorentzon, J.; Fulscher, M. P.; Roos, B. J. Am. Chem. Soc. 1995 117,9265 .

(29) Fulscher, M. P.; Serrano-Andres, L.; Roos, B. J. Am. Chem. Soc. 1997, 119, 6168.

(30) Fulscher, M. P.; Roos, B. J. Am. Chem. Soc. 1995, 117, 2089.

(31) Shukla, M. K.; Leszczynski, J. J. Phys. Chem. A 2002, 106, 1011.

(32) Shukla, M. K.; Leszczynski, J. J. Phys. Chem. A 2002, 106, 4709.

(33) Sobolewski, A.; Domcke, W. Phys. Chem. Chem. Phys. 2004, 6, 2763.

(34) Wesoloswki, T. J. Am. Chem. Soc. 2004, 126, 11444

(35) Jean, J. M.; Hall, K. B. Proc. Natl. Acad. Sci. U.S.A. 2001, 98, 37.

(36) Jean, J. M.; Hall, K. B. Biochemistry 2002, 41, 13152.

(37) Mennucci, B.; Tonniolo, A.; Tommasi, J. J. Phys. Chem. A 2001, 105,4749

(38) Mishra, S. K.; Shukla, M. K.; Mishra, P. C. Spectrochim. Acta, Part A 2000, 56, 1355.

(39) Shukla, M. K.; Mishra, S. K.; Kumar, A.; Mishra, P. C. J. Comput. Chem. 2000, 21, 826.

(40) Shukla, M. K.; Leszczynski, J. J. Phys. Chem. A 2002, 106, 11338.

(41) Marques, M. A. L.; Castro, A.; Bertsch, G. F.; Rubio, A. Comput. Phys. Commun. 2003, 151, 60; available at http://www.tddft.org/programs/ octopus

(42) Castro, A.; Marques, M. A. L.; Alonso, J.; Rubio, A. J. Comput. Theor. Nanosci. 2004, 1, 230

(43) Yabana, K.; Bertsch, G. F. Phys. Rev. B 1996, 54, 4484.

(44) Yabana, K.; Bertsch, G. F. Int. J. Quantum Chem. 1999, 75, 55.

(45) Yabana, K.; Bertsch, G. F. Phys. Rev. A 1999, 60, 3809.

(46) Rubio, A.; Alonso, J.; Blase, X.; Balbas, L.; Louie, S. Phys. Rev. Lett. 1996, 77, 247.

(47) Rubio, A.; Alonso, J.; Blase, X.; Louie, S. Int. J. Mod. Phys. B 1997, 11, 2727

(48) Casida, E. In Recent Advances in Density Functional Methods Part 1; Chong D. P., Ed.; World Scientific: Singapore, 1995; p 115.

(49) Perdew, J.; Zunger, A. Phys. Rev. B 1981, 23, 5048. 3865 .

(50) Perdew, J. P.; Burke, K.; Ernzerhof, M. Phys. Rev. Lett. 1996, 77,

(51) Marques, M. A. L.; Castro, A.; Rubio, A. J. Chem. Phys. 2001, 115,3006

(52) Despite the equivalence encountered in the photoabsorption crosssection of gas-phase guanine by using these two functionals, which is sufficient for our purposes to justify the LDA option, one has to be extremely careful in general in the choice of $v_{\mathrm{xc}}$. For instance, it was shown that in processes that involve charge-transfer mechanisms, such as fluorescence in base pairs, the correct asymptotic behavior of the exchange-correlation functional may affect the accuracy of the outcome. See, e.g., Grimme, S.; Parac, M. ChemPhysChem 2003, 4, 292.

(53) Troullier, N.; Martins, J. L. 1991, 43, 1993.

(54) Arnott, S.; Hukins, D. W. L. Biochem. Biophys. Res. Commun. 1972 47, 1504.

(55) Gao, Y. G.; Robinson, H.; Wang, A. H. Eur. J. Biochem. 1999, $261,413$.

(56) Brunger, A. T.; Karplus, M. Proteins 1988, 4, 148.

(57) Brooks, B. R.; Bruccoleri, R. E.; Olafson, B. D.; States, D. J.; Swaminathan, S.; Karplus, M. J. Comput. Chem. 1983, 4, 187.

(58) MacKerrel, A. D.; Bashford, D.; Bellott, M. Dunbrack, R. L.; Evanseck, J. D.; Field, M. J.; Fischer, S.; Gao, J.; Huo, H.; Ha, S.; JosephMcCarthy, D.; Kuchnir, L.; Kuczera, K.; Lau, F. T. K.; Mattos, C. Michnick, S. Straub, T.; Watanabe, M.; Wiorkiewicz-Kuczera, J.; Yin, D.; Karplus, M. J. Phys. Chem. B 1998, 102, 3586.

(59) Perdew, J. P.; Wang, Y. Phys. Rev. B 1992, 45, 13244.

(60) Zhang, H.; Di Felice, R., unpublished.

(61) Calzolari, A.; Di Felice, R.; Molinari, E.; Garbesi, A. J. Phys. Chem. B 2004, 108, 2509.

(62) Calzolari, A.; Di Felice, R.; Molinari, E.; Garbesi, A. J. Phys. Chem. B 2004, 108, 13058.

(63) Shukla, M. K.; Mishra, S. K. Chem. Phys. 1999, 240, 319.

(64) Merchan, M.; Serrano-Andres, L. J. Am. Chem. Soc. 2003, 125, 8108.

(65) Ismail, N.; Blancafort, L.; Olivucci, M.; Kohler, B.; Robb, M. A. J. Am. Chem. Soc. 2002, 87, 6818.

(66) Clark, L. B.; Tinoco, I. J. Am. Chem. Soc. 1965, 87, 11.

(67) Miles, D. W.; Robins, R. K.; Eyring, K. Proc. Natl. Acad. Sci. U.S.A. 1967, 57, 1139

(68) Sprecher, C. A.; Johnson, W. C., Jr. Biopolymers 1977, 16, 2243. (69) Voelter, W.; Records, R.; Bunnenberg, E.; Djerassi, C. J. Am. Chem. Soc. 1968, 90, 6163 .

(70) Fucaloro, A. F.; Forster, L. S. J. Am. Chem. Soc. 1971, 93, 6443

(71) Eaton, W. A.; Lewis, T. P. J. Chem. Phys. 1970, 53, 2164.

(72) Stewart, R. F.; Davidson, N. J. Chem. Phys. 1963, 39, 255.

(73) Stewart, R. F.; Davidson, N. Biopolymers 1964, 1, 465

(74) Kim, N. J.; Jeong, G.; Kim, Y. S.; Sung, J.; Kim, S. K.; Park, Y. J. Chem. Phys. 2000, 113, 10051.

(75) Sobolewski, A.; Domcke, W. Eur. Phys. J. D 2002, 20, 369.

(76) Holmen, A.; Broo, A.; Albinsson, B.; Norden, B. J. Am. Chem. Soc. 1997, 119, 12240.

(77) Broo, A. J. Phys. Chem. A 1998, 102, 526.

(78) Callis, P. R.; Rosa, E. J.; Simpson, W. T. J. Am. Chem. Soc. 1964, 86, 2292.

(79) Clark, L. B. J. Phys. Chem. 1995, 99, 4466

(80) Brunner, W. C.; Maestre, M. F. Biopolymers 1975, 14, 555.

(81) Hernández, C. E. C.; Marai, C.; Kohler, B., unpublished, 2004

(82) Nir, E.; Muller, M.; Grace, L. I.; de Vries, M. S. Chem. Phys. Lett. 2002, 59, 355.

(83) Mons, M.; Dimicoli, I.; Piuzzi, F.; Tardivel, B.; Elhanine, M. $J$. Phys. Chem. A 2002, 106, 5088 .

(84) Jortner, J.; Bixon, M.; Langenbacher, T.; Michel-Beyerle, M. E. Proc. Natl. Acad. Sci. U.S.A. 1998, 95, 12759.

(85) Dreuw, A.; Weisman, J. L.; Head-Gordon, M. J. Chem. Phys. 2003 , 119,2943

(86) Dreuw, A.; Weisman, J. L.; Head-Gordon, M. J. Am. Chem. Soc. 2004, 126, 4007

(87) Gritsenko, O.; Baerends E. J. J. Chem. Phys. 2004, 121, 655.

(88) Brealey, G.; Kasha, M. J. Am. Chem. Soc. 1955, 77, 4462.

(89) Callis, P. R. Annu. Rev. Phys. Chem. 1983, 37, 691.

(90) With this reasoning, we do not claim that the values of the $\pi \pi^{*}$ transitions are to be considered exact also for the solution phase, but they are not expected to undergo major modifications in different solutions. Most likely, the effect of the environment on the $\pi \pi^{*}$ transitions can be expected to be a systematic shift toward low energies, which should be taken into account for a more strict comparison in Tables 1-6. However, such a shift cannot be computed at present at the same level of theory.

(91) Varsano, D.; Di Felice, R.; Marques, M. A. L.; Rubio, A., unpublished. 